\title{
Studienstart zur Typ-2-Diabetestherapie Kann frühe Kombi mit DPP-4-Hemmer den Wirkverlust stoppen?
}

- Die orale Diabetestherapie verliert mit den Jahren oft rasch an Wirkung. Lässt sich die progrediente Verschlechterung der Blutzuckerkontrolle mit neueren Wirkstoffen und veränderten Therapiestrategien aufhalten? In einer großen Studie soll dies nun geklärt werden.

In der UKPD-Studie mit neu diagnostizierten Typ-2-Diabetikern erreichte schon nach drei Jahren jeder zweite mit der anfänglichen Monotherapie nicht mehr das Therapieziel. Die rasche Progredienz wird vor allem auf einen zunehmenden Verlust der Betazellfunktion zurückgeführt. Es gibt Hinweise aufgrund von tierexperimentellen Daten und Surrogatparametern aus klinischen Studien, dass DPP4-Hemmer über eine Erhöhung der Inkretinspiegel die Betazellfunktion günstig beeinflussen könnten. Wissenschaftler hoffen, dass vielleicht eine sehr frühzeitige Kombitherapie - ähnlich wie sie sich immer mehr in der Bluthochdruckbehandlung etabliert das Fortschreiten der Erkrankung aufhalten kann. Beide Strategien sollen nun in der VERIFY* ${ }^{\star}$-Studie geprüft werden. Sie wurde beim US-Diabeteskongress in Philadelphia vorgestellt. Für die vom Unternehmen Novartis unterstützte Untersuchung wurden mindestens 2000 Typ-2-Diabetespatienten innerhalb der ersten zwei Jahre nach Diagnose rekrutiert. Diese erhalten randomisiert und doppelblind entweder den DPP-4-Hemmer Vildagliptin plus Metformin (Eucreas ${ }^{\circledast}$ ) oder Metformin allein. Primärer Endpunkt der Studie ist die Zeit bis zum „Therapieversagen" - definiert als $\mathrm{HbA}_{1 \mathrm{c}}$-Wert über $7 \%$ in zwei Folgeuntersuchungen. Kann die frühe Kombination mit dem Gliptin den progredienten Verlust der Blutzuckerkontrolle aufhalten?

Die Studie findet in 260 Zentren in 30 Ländern statt. Sie wird voraussichtlich etwa fünf Jahre dauern. Gestartet wird sie derzeit, die Ergebnisse werden etwa im Jahr 2019 erwartet. bös

* Vildagliptin Efficacy in combination with MetfoRmln For earlY treatment of type 2 diabetes

Quelle: ADA-Kongress Philadelphia, 8.-12. Juni 2012

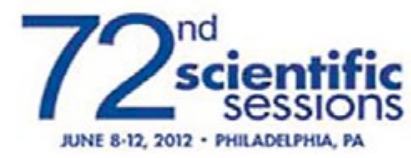

\section{Direktvergleich DPP-4-Hemmer mit Sulfonylharnstoff Mit Gliptin bleiben Gewichtszunahme und Hypoglykämien öfter aus}

- Oft wird bei Typ-2-Diabetikern zur Blutzuckerkontrolle zum Metformin ein Sulfonylharnstoff gegeben. Dies führt jedoch in vielen Fällen zu Hyperglykämien und Gewichtszunahme. Eine beim US-Diabeteskongress der ADA in Philadelphia vorgestellte Post-hoc-Analyse ergab, dass diese Nebenwirkungen seltener auftreten, wenn Metformin mit dem DPP-4-Hemmer Linagliptin kombiniert wird.

In der 2-jährigen Post-hoc-Analyse einer PhaseIII-Studie wurde untersucht, wie viele der erwachsenen Patienten mit Typ-2-Diabetes bei Einnahme von Metformin plus Linagliptin (Trajenta ${ }^{\circledR}$ ) bzw. Glimepirid einen $\mathrm{HbA}_{1 \mathrm{c}}$ von $<7 \%$ ohne Gewichtszunahme und ohne Hypoglykämie erreichten [1]. Die Gewichtszunahme war dabei definiert als $<1 \mathrm{~kg} \mathrm{Zu-}$ nahme gegenüber dem Ausgangswert, die Hypoglykämie als Ereignis pro Protokoll. Der Ausgangs$\mathrm{HbA}_{1 \mathrm{c}}$-Wert war in beiden Gruppen ähnlich (Linagliptin 7,2\%; Glimepirid 7,3\%).

Dabei erhielten 504 hyperglykämische Patienten während eines Zeitraums von 104 Wochen zusätzlich zu Metformin randomisiert $5 \mathrm{mg} / \mathrm{d}$ Linagliptin $(\mathrm{n}=233)$ oder $1-4 \mathrm{mg} / \mathrm{d}$ Glimepirid $(\mathrm{n}=271)$. Das
Ergebnis: Die Blutzuckersenkung war in beiden Gruppen ähnlich, 76\% der Patienten erreichten einen Ziel-HbA $1 c-$ Wert $<7 \%$. Mit Linagliptin erreichten aber deutlich mehr Patienten, nämlich 54\%, den kombinierten Endpunkt (Ziel-HbA $\mathrm{Hc}_{\mathrm{c}}$ von $<7 \%$ ohne Gewichtszunahme oder Hypoglykämie) als $\mathrm{Pa}$ tienten, die den Sulfonylharnstoff bekamen, dort waren es 23\%. Das Chancenverhältnis zum Erreichen des kombinierten Endpunkts war mit Linagliptin 3,9-mal höher $(\mathrm{p}<0,0001)$.

Auch hinsichtlich der Nebenwirkungen profitierten die Patienten in der Linagliptin-Gruppe: $\mathrm{Zu}$ einer Hypoglykämie kam es mit dem DPP-4-Hemmer bei 6\% der Patienten, mit dem Sulfonylharnstoff bei $42 \%$. Eine Gewichtszunahme mit dem Gliptin wurde bei $22 \%$ der Patienten beobachtet, mit Glimepirid waren es deutlich mehr, nämlich 55\%.

Linagliptin ist in Europa seit etwa einem Jahr zugelassen, aber derzeit nicht im Handel erhältlich.

wer

Quelle: Nach Informationen der Unternehmen Boehringer Ingelheim und Lilly beim ADA-Kongress, 9.6.2012, Philadelphia, USA

\footnotetext{
1. Gallwitz B, Rosenstock J et al. Linagliptin is More Effective than Glimepiride at Achieving a Composite Outcome of A1C Target with No Hypoglycemia and No Weight Gain over two Years in Mildly Hyperglycemic Type 2 Diabetes Patients on Metformin. Abstract P-1044,
} 